\title{
DOENÇA DE ALZHEIMER: ASPECTOS FISIOPATOLÓGICOS, QUALIDADE DE VIDA, ESTRATEGIAS TERAPÊUTICAS DA FISIOTERAPIA E BIOMEDICINA.
}

\author{
Alzheimer's Disease: physiopathological aspects, quality of life, therapeutic \\ strategies of physiotherapy and biomedicine.
}

Eduarda Machado Bitencourt ${ }^{1}$, Claudia Marlaine Xavier Kuerten², Josiane Budny², Talita Tuon ${ }^{3}$

Autor $^{1}$, Autor ${ }^{1}$, Autor ${ }^{1,3}$, Autor ${ }^{1,2,3}$.

${ }^{1}$ Bem Viver Com Alzheimer, Universidade do Extremo Sul Catarinense (UNESC), Unidade Acadêmica de Ciências da Saúde, Curso de Fisioterapia.

²Laboratório de Sinalização Neural e Psicofarmacologia, Laboratório de Neurotoxicidade e Neuroproteção, Programa de Pós-Graduação em Ciências da Saúde da UNESC.

${ }^{3}$ Unidade de Doenças Neurodegenerativas, Laboratório de Neurociências, Programa de Pós-Graduação em Ciências da Saúde da UNESC.

\section{Endereço para correspondência:}

Autor

Universidade do Extremo Sul Catarinense (UNESC), Unidade Acadêmica da Saúde, Curso de Fisioterapia.

Avenida Universitária, 1105, Bairro Universitário, Criciúma-SC, Brasil. CEP: 88806000

Email: talitatuon@gmail.com 


\title{
Resumo
}

Com aumento da expectativa de vida os indivíduos vem alcançando idade mais avançada e desenvolvendo doenças neurodegenerativas como a doença de Alzheimer. Uma doença que leva danos cognitivos, proprioceptivas e a demência. Se apresenta em três estágios: Estagio 1: No inicio da doença com problemas moderados de memória como esquecimento; Estagio 2: o indivíduos necessita de cuidados ocorre perda da funcionalidade, temporalidade e esquecimentos, perda de memória recentes, a interpretação dos estímulos é prejudicada. Não consegue mais realizar suas atividades diárias sozinho. Estagio 3: perda quase completa da capacidades cognitiva, completa dependência de um cuidador. Métodos Foram realizadas buscas sistematizadas em bases de dados eletrônicas (Scielo, Pubmed, Google Acadêmico). Resultados e conclusão: Os estudos analisados sugerem a eficácia da Fisioterapia, na melhora da função motora, para manter o equilíbrio, a força e a cognição em pacientes com DA. Além disso, exercício está associado com menor prevalência e incidência de demência (32\%), bem como de declínio cognitivo. a Biomedicina é muito importante para auxiliar nas áreas que envolvam avaliações da qualidade de vida deste paciente, criar estratégias educativas, de prevenção, promoção e intervenção na busca de uma velhice bem-sucedida junto aos familiares e cuidadores.

Palavras-chave: Doença de Alzheimer, Fisioterapia na Doença de Alzheimer, Biomedicina na Doença de Alzheimer, Tratamento para Doença de Alzheime

\begin{abstract}
With increasing life expectancy individuals come with more advanced age and developing neurodegenerative diseases like Alzheimer's disease. A disease that leads to cognitive, proprioceptive and dementia damage. If present in three stages: Stage 1: At the beginning of the disease with moderate memory problems such as
\end{abstract}


forgetfulness; Stage 2: the need for health care and loss of functionality, temporality and forgetfulness, recent memory loss, an interpretation of the stimuli is impaired. You can not do your daily activities alone. Stage 3: almost complete loss of cognitive ability, complete dependence on a caregiver. Methods Systematized searches were performed in electronic databases (Scielo, Pubmed, Google Scholar). Results and conclusion: The studies analyzed suggest the efficacy of physical therapy, the improvement of motor function, to maintain balance, strength and cognition in patients with $\mathrm{AD}$. In addition, exercise is associated with lower prevalence and incidence of dementia (32\%) as well as cognitive decline. Biomedicine is very important for areas that involve evaluations of the patient's quality of life, creating educational strategies, prevention, promotion and intervention in the search for a successful old age among family members and caregivers.

Key words: Alzheimer's Disease, Alzheimer's Disease Physiotherapy, Alzheimer's Disease Biomedicine, Treatment for Alzheimer's Disease.

\section{INTRODUÇÃO}

Com aumento da longevidade humana e a melhor expectativa de vida boa parte dos indivíduos vem alcançando idade mais avançada e junto a ela o desenvolvimento de doenças neurodegenerativas como a doença de Alzheimer ${ }^{1,2}$.

A doença de Alzheimer é uma neuropatologia que leva deterioração das células cerebrais e que se agrava ao longo do tempo agindo de forma progressiva, irreversível e insidiosa, o que leva a demência, perda de memória e diversos outros danos cognitivos ${ }^{3}$.

A DA caracteriza-se principalmente pela perda de memória e quadro demencial comprometendo fatos recentes, distúrbios psíquicos que levam a mudanças de personalidade em relação as suas ideias, julgamentos e linguagem o que compromete suas relações sociais, interpessoais e no trabalho ${ }^{4}$. 
Neuropatologicamente, as principais lesões cerebrais, a presença de inúmeras placas senis com depósitos extracelulares de proteína beta amilóide (APP) e emaranhados neurofibrilar composto de proteína TAU. Esta condição leva a alterações que dificultam a busca por tratamento eficaz e incapacidade de cuidar de si própria ${ }^{5}$.

A demência da DA não se apresenta de forma igual para todos os indivíduos, existem inúmeros fatores de riscos envolvidos formando complexo de sinais e sintomas que progridem de forma lenta. Portanto, algumas pessoas são mais propensas a DA do que outras, não há somente uma única causa, porém existe um padrão de evolução da doença, o qual se apresenta em três estágios ${ }^{3,6}$

Estagio 1: caracterizado pelo inicio da doença com aparecimento dos sintomas, problemas moderados de memória como esquecimento de números de telefone, nomes, porém por serem sutis não são imediatamente detectáveis. Dificuldades em manter atenção e desorientações no espaço são bem evidentes neste estagio ${ }^{3,6}$.

Estagio 2: nesta fase o indivíduos necessita de cuidados e supervisão para determinadas necessidades, ocorre perda da funcionalidade, temporalidade e esquecimento para recordações recentes embora a memória para acontecimentos passados permaneça intacta. A capacidade de interpretar os estímulos (tato, paladar, visão, audição) é prejudicada. Além disso, problemas com insônia devido a incapacidade de distinguir o dia da noite. $O$ indivíduo não consegue mais realizar suas atividades diárias sozinho, pois seus movimentos são menos precisos e coordenados. As mudanças bruscas de humor são constantes, o que torna o portador de DA agressivo e agitado e sempre repetindo as mesmas frases ${ }^{3,6}$.

Estagio 3: 0 indivíduos com DA nesta fase tem perdido quase que por completo suas capacidades cognitivas, tornando-se completamente dependente de um familiar ou cuidador externo, já não se tem mais a sua capacidade psicomotora ${ }^{3,6}$. 


\section{METODOLOGIA}

O presente estudo trata-se de uma revisão de literatura, que busca abordar em um primeiro momento, os aspectos fisiopatológicos da Doença de Alzheimer e o tratamento nas diversas áreas. Em um segundo momento, o tratamento relacionados à prática fisioterapêutica, e a participação da Biomedicina no desenvolvimento de estratégias e orientações para com os indivíduos com a Doença de Alzheimer e seus familiares. As palavras-chaves: Doença de Alzheimer, Fisioterapia na Doença de Alzheimer, Biomedicina na Doença de Alzheimer, Tratamento para Doença de Alzheimer. Foram usadas em bases nacionais e internacionais, sugerindo suas respectivas traduções. Em bases de dados como: Scielo, LILACS e PubMed.

\section{FISIOPATOLOGIA E ETIOLOGIA}

A etiologia da $A D$ ainda não foi completamente elucidada, mas há evidências de que esta doença neurodegenerativa é uma síndrome multifatorial, resultante de um conjunto complexo de fatores neuroquímicos. Estudos genéticos humanos e em modelos animais transgênicos da DA sugerem que vários eventos desencadeiam um caminho patobiológico convergente levando ao mau funcionamento e morte celular?.

A Doença de Alzheimer é caracterizada por uma série de alterações neuropatológicas, que resultam em perda de memória, confusão, afetação do julgamento, desorientação e problemas na expressão. Os sintomas tendem a piorar ao longo do tempo ${ }^{4}$. Dentre essas alterações a atrofia do hipocampo e do córtex cerebral, com envolvimento primário do córtex de associação fronto-temporal. A atrofia acontece devido a dilatação dos ventrículos laterais principalmente do corno temporal ${ }^{8,9}$.

Quando analisados microscopicamente, as características neuropatológicas da doença são a presença de placas extracelulares de beta amilóide, os emaranhados neurofibrilares intracelulares, que resultam da hiperfosforilação 
anormal da proteína tau, e uma extensa perda neuronal. A proteína tau dá estabilidade aos microtubulos na célula neuronal e facilitam a polimerização da tubulina para que assim os microtubulos sejam formados 8,9 .

A hiperfosforilação impede a sua função normal e por vez facilita sua agregação em fibrilas. O efeito destes eventos é a alteração de da estrutura dos microtubulos que junto com o empacotamento da proteína tau provoca implicações no mecanismo de transporte neuronal ${ }^{10,11}$.

Nas paredes dos vasos sanguíneos no parênquima cerebral há presença de depósitos fibrilares amiloidais, acúmulo de filamentos anormais de proteína Tau que levam a formação de novelos neurofibrilares gerando processo inflamatório, perdas neuronais e sinápticas ${ }^{12}$.

A proteína precursora de amilase (APP) é uma proteína intermembrana encontrada em pacientes com ou sem a doença. Porém em indivíduos com DA a clivagem da APP ocorre de modo errôneo, isto leva formação de peptídeo insolúvel, que possui uma porção hidrofóbica com potencial agregatório o que leva a formação de um grumo que gera inflamação, levando morte do neurônio ${ }^{4}$. Isto pode desencadear a hiperfosforilação da proteína tau. Assim estes APP não funcionais quando reunidos formam emaranhados que interferem na função neuronal, tornamse tóxicos e levam a formação de placas beta amilóides. Estes emaranhados atingem o neurônio quando a proteína tau modifica-se. O microtubulo é ligado por tubulinas que é a proteína tau. A tau modificada na DA separa os fios de proteínas dos microtubulos, assim estes fios dispersos unem-se e aglomeram-se no neurônio prejudicando as sinapses com morte de célula neuronal. Sendo este um processo tóxico, continuo que acomete o cérebro com atrofia cerebral. Acredita se que fatores genéticos e estilo de vida colaboram para surgimento da $\mathrm{DA}^{13,14,15,16}$.

\section{EPIDEMIOLOGIA DA DOENÇA DE ALZHEIMER}

Em abril de 2012, a OMS publicou o documento "Demência: Uma Questão de Saúde Pública", demonstrando grande preocupação com o problema que afeta a qualidade de vida das pessoas idosas, principalmente nos países em 
desenvolvimento. Estimam-se em 35,6 milhões o número de pessoas com demência em 2010, projetando uma duplicação neste número a cada 20 anos; 65,7 milhões em 2030 e 115,4 milhões em 2050. O numero de novos casos de demência a cada ano no mundo é de cerca de 7,7 milhões, aproximadamente uma pessoa diagnosticada a cada quatro segundos ${ }^{17}$.

A DA é o tipo de demência mais comum no mundo. A prevalência de Alzheimer em pessoas com mais de 65 anos é de 1,6\% na África, 3,9\% no Leste Europeu, 4,0\% na China, 5,4\% no Oeste Europeu, e 6,4\% na América do Norte ${ }^{18}$. $\mathrm{Na}$ Europa como um todo, $6 \%$ das pessoas com mais de 65 anos e $30 \%$ das pessoas com mais de 90 anos têm algum tipo de demência ${ }^{19}$.

No Brasil, em estudo populacional realizado em um município de Catanduva, do Estado de São Paulo com aproximadamente 100 mil habitante, $25 \%$ dos idosos foram avaliados, encontrando prevalência de demência semelhante à literatura. Foi observaram prevalência de $7,1 \%$ de casos de demência (118 habitantes) em uma população de 1.656 indivíduos com idade igual ou maior que 65 anos. A taxa de incidência anual foi de 7,7 casos por 100.000 habitantes. Em Catanduva, 55,1\% dos casos de demência receberam este diagnóstico ${ }^{20}$.

A prevalência da demência aumenta com o envelhecimento, a idade é o maior fator de risco para a doença. Entre 60 e 64 anos apresenta prevalência de $0,7 \%$, passando por $5,6 \%$ entre 70 e 79 anos, e chegando a $38,6 \%$ nos 90 anos. A partir dos 65 anos, sua prevalência dobra a cada cinco anos. A incidência não diminui mesmo na faixa etária, acima dos 95 anos. Alterações genéticas também podem ser responsáveis por aumentar o risco da demência de Alzheimer, mesmo que em menor incidência. As mutações do gene da proteína precursora do amilóide (cromossomo 21), dos genes das présenilinas 1 e 2 (cromossomos 14 e 1, respectiva- mente), também o polimorfismo da apolipoproteína $E$ (cromossomo 19) são exemplos de alterações genéticas que podem aumentar o risco para DA ${ }^{20}$.

A prevalência das demências e influenciada por outras variáveis demográficas, como escolaridade e nível socioeconômico. Em um estudo realizado 
na comunidade de imigrantes latinos que vivem na Califórnia foi observado que nível educacional e cultura anglo estavam associados ao baixo risco de demência. E o risco para desenvolver demência foi aumentado em oito vezes para idosos com diabetes mellitus tipo $2{ }^{21}$.

No Brasil o baixo nível de escolaridade da população exige, inclusive, variações no ponto de corte utilizadas nos instrumentos para rastreio e diagnostico de demência. Em relação ao gênero, alguns estudos encontraram uma maior prevalência de demência entre as mulheres. Porém quando estes pesquisadores analisaram estatisticamente esta diferença, observaram que não existe predomínio no gênero feminino. Uma possível explicação para o fato de que existem mais mulheres acometidas com demência de Alzheimer e a maior expectativa de vida do gênero feminino, e não algum fator de risco específico relacionado ao sexo 22.

Os aspectos genéticos, com histórico familiar de demência de Alzheimer aumenta o risco para o desenvolvimento da DA em cerca de quatro vezes. Estudos demonstram que $75 \%$ dos casos de DA possuem histórico familiar positivo para a síndrome ${ }^{22}$.

As Nações Unidas após ter aplicado estimativas de prevalência específicas relativas à idade, ou idade e sexo, às projeções de população. Estima-se que o número duplique a cada 20 anos, atingindo os 65,7 milhões em 2030 e os 115,4 milhões em 2050. Grande parte desse aumento pode ser claramente atribuído ao aumento do número de pessoas com demência em países de baixo e médio rendimento. Em 2030 63,4\% de todas as pessoas com demência deverão viver em países de baixo e médio aumentando para $70,5 \%$ em 2050. As estimativas atualizadas para 2020 (48,1 milhões) e para 2040 (90,3 milhões) ${ }^{22}$.

\section{DIAGNOSTICO DA DOENÇA DE ALZHEIMER}

A busca inicial por orientações sobre a doença de Alzheimer é adiada devido os primeiros sintomas de a doença serem confundidos com processos naturais do envelhecimento. Isto faz com que a doença seja diagnosticada tardiamente ${ }^{10}$. 
$\mathrm{Na}$ DA podem ser encontradas disfunções de estruturas neurovasculares, disfunções mitocondriais, processos inflamatórios e também estresse oxidativo. A confirmação do diagnóstico se dá somente por exame microscópico do tecido cerebral através de cortes histológicos após óbito do paciente. Temos somente o diagnóstico da doença clínica com início lento dos sintomas e progressivo perdas das funções cerebrais. Consequentemente tem-se uma eminente neurodegeneração cerebral. Não há exames laboratoriais complementares capazes de confirmar a DA, sua avaliação clinica é de suma importância ${ }^{23}$.

Exames clínicos, físicos, laboratoriais e de neuroimagem precisam ser realizados para que haja diferenciação de outras doenças. Testes psicológicos para verificar o funcionamento cognitivo, anamnese completa e detalhada com todo histórico do paciente, presença de doenças preexistentes, exposição ambiental, contato com agentes tóxicos ${ }^{24}$. Estas informações além de outros exames, quando analisados permite o medico fazer associação dos dados da historia com observação do comportamento do paciente e assim identificar a intensidade de perdas e hipóteses que indicam a presença da doença $20,25,1,2,26$.

Em casos de suspeita clínica da doença, os exames complementares são: eletroencefalograma; dosagem sérica de drogas; HIV; vitaminas B1 (tiamina) e B3 (niacina); screening de metais pesados (zinco, cobre, mercúrio e manganês); infecção terciária por Borélia - doença de Lyme; lúpus eritematoso disseminado (anticorpos antinucleares e complemento); gasometria; marcadores neoplásicos; homocisteinemia; cisternografia isotópica; biópsia cerebral ${ }^{23}$.

Marcadores moleculares auxiliam no diagnóstico precoce da DA quando relacionados ao metabolismo neuronal e inflamação, o que contribui na avaliação dos mecanismos de degeneração quando comparados ao declínio cognitivo ${ }^{23}$.

Os níveis aumentados da proteína Tau e a concentração reduzida da beta amiloide demonstram-se marcadores biológicos presentes na investigação clinica da doença. Porem essa analise tem sido realizada somente na área da pesquisa. Os indícios sugerem que a deposição da proteína beta-amilóide na formação das placas neurítica e da proteína TAU nos novelos neurofibrilares estao diretamente ligados á lesão neuronal. A análise no LCR (líquido cefalorraquidiano) em indivíduos 
com DA exibe níveis baixos do peptídeo A-beta 42 característico da patologia e também por sua deficiente fagocitose, pois portadores de DA apresentam uma drenagem fisiológica prejudicada quando comparados a indivíduos não dementes ${ }^{24}$.

O marcador por imagem mais especifico da DA é a atrofia encontrada na região temporal medial onde são observadas muitas alterações na substancia branca e que podem ser analisadas através Imagem de ressonância magnética (IRM). Já marcadores genéticos relacionados à DA tem-se o alelo APO E que é principal apolipoproteína encontrada no cérebro, localizado no braço longo do cromossomo 19. Estudos com indivíduos com DA demonstraram alterações no cromossomo 19. O gene Apo E contribuem na formação da proteína Apoliproteina $\mathrm{E}$, e esta combina-se com gordura do organismo ${ }^{24}$.

Para análise cognitiva na investigação de demências, podem ser utilizados testes neuropsicológicos. Quando a suspeita recai sobre a DA, o paciente é submetido a uma série de testes cognitivos, como o Mini Exame do Estado Mental (MEEM) e exames de neuroimagem para um diagnóstico de provável doença de Alzheimer, por exclusão de outras doenças senis rastreando as perdas cognitivas 20,26 .

Conhecer a fisiopatologia da DA é importante para o desenvolvimento de biomarcadores que contribuem no diagnóstico precoce e na busca de métodos terapêuticos que visem à origem da doença. Não utilizados na rotina laboratorial, somente em ensaios pré clínicos os, os biomarcadores positivos como liquido cefalorraquidiano com redução da $A \beta 42$ e elevação da proteína Tau e fosfo-Tau demonstram ser um método sensível e específico no diagnostico precoce da DA ${ }^{26}$, 20. O diagnóstico definitivo DA pode ser feito somente por autopsia do cérebro do paciente. Nessa avaliação neuropatológica revela-se bruta atrofia cerebral, significando perda de neurônios ${ }^{27}$.

\section{TRATAMENTO MEDICAMENTOSO}

As regiões cerebrais associadas às funções mentais superiores, como o córtex frontal e o hipocampo, são as mais comprometidas pelas alterações bioquímicas decorrentes da DA. Em nível celular associada a DA temos a redução 
dos níveis de Acetilcolina. Com o déficit de acetilcolina tem-se a perda de memória e desta forma o tratamento da DA é voltado para inibição da acetilcolinesterase, pois assim haverá quantidades significativas do neurotransmissor acetilcolina na fenda sináptica, fazendo o paciente apresentar melhoras na memória e compensará o déficit colinérgico ${ }^{28,29}$. É o caso do fármaco de primeira escolha no tratamento de DA, o Donepezil, que exerce sua atividade terapêutica inibindo reversivelmente a acetilcolinesterase o que melhora o perfil cognitivo da doença e apresenta menor toxicidade em relação a outros fármacos inibidores de acetilcolinesterase ${ }^{30,31}$.

Os fármacos como Tacrina, Rivastigmina, Galantamina considerados de primeira linha e o Donezepil possuem propriedades farmacológicas levemente diferentes, mas todas apresentam eficácia sobre a função cognitiva e atividades diárias, pois possuem potencial inibidor de acetilcolinesterase. Porém no Brasil, o fármaco aprovado e que somente pode ser utilizado no estagio avançado da DA é o Donepezil, os outros fármacos ainda não tiveram autorização das agencias reguladoras. Ainda não existe a cura para doença de Alzheimer, mas a utilização dos fármacos busca amenizar os efeitos da doença, para que ela não progrida $29,32,12$.

Outro fármaco também utilizado no tratamento da DA é chamado Memantina. Este fármaco é um antagonista de receptores NMDA envolvidos na transmissão dos sinais nervosos em áreas ligadas a memoria e aprendizagem bloqueando os canais iônicos, melhorando a transmissão dos sinais nervosos e a memória ${ }^{33,34}$.

\section{QUALIDADE DE VIDA}

Segundo a OMS, Qualidade de Vida como a percepção do indivíduo sobre sua posição na vida, no contexto da cultura e sistema de valores nos quais ele vive e em relação aos seus objetivos, expectativas envelhecem, padrões e preocupações $^{35}$.

O envelhecer com ou sem patologia depende de fatores biológicos, psicológicos e sociais, ou seja, as pessoas envelhecem de formas diferentes de acordo com os seus estilos de vida. Não devemos considerar o envelhecimento 
como sinônimo de doença, pois é possível envelhecer com saúde e boa qualidade de vida. Em idosos com DA os danos cognitivos causados geram sentimentos de desamparo, fragilidade e falta de perspectiva para o futuro. Os processos degenerativos levam a abalos psíquicos e funcionais que comprometem a qualidade de vida ${ }^{36,35}$.

As práticas médicas e os tratamentos farmacológicos buscam retardar a evolução da doença, o que contribuem na discreta melhora da capacidade de autonomia do idoso com DA, trazendo melhoras no seu estado de bem-estar e qualidade vida. Sabe-se o quão importante na busca do bem-estar do idoso com DA é também o trabalho transdisciplinar em conjunto com diversas áreas da saúde como nutrição, fisioterapia, psicologia, farmácia, enfermagem, biomedicina trazendo resultados positivos no processo saúde-doença ${ }^{35,} 37$.

No tratamento do paciente com doença de Alzheimer, medidas não farmacológicas como a estimulação de pacientes apáticos, a melhor estruturação do tempo, da ocupação e do meio ambiente do paciente, elaboração de programas para o cotidiano e o desenvolvimento de rotinas contribuem para seu bem estar ${ }^{38,} 39$.

O exercício físico tem demonstrado ação benéfica como um tratamento não farmacológico para a doença de Alzheimer, o que resulta em melhora na função cognitiva, maior equilíbrio, redução nos distúrbios de comportamento e no risco de quedas e também melhora na função motora de pacientes com doença de Alzheimer. A inclusão de diversas áreas da saúde possibilita maior atendimento $e$ entendimento das necessidades do idoso com DA, não esquecendo que o tratamento não medicamentoso da doença de Alzheimer é dirigido não apenas ao paciente, como também aos seus familiares e cuidadores ${ }^{40,37}$.

Orientações sobre as formas evolutivas da doença, as maneiras de como lidar com as mudanças comportamentais do idoso com DA, além das adaptações necessárias no ambiente são medidas necessárias para que a família, cuidadores e o próprio idoso consigam obter melhor qualidade de vida e bem-estar ${ }^{41,36}$. 


\section{TRATAMENTO FISIOTERAPEUTICO E PREVENÇÃO BIOMEDICA}

A doença de Alzheimer se manifesta lentamente com progressiva deterioração, que prejudica o paciente em suas atividades diárias AVD's e em seu desempenho social, tornando um ser cada vez mais dependente de cuidados ${ }^{4}$. Apesar de não existir um tratamento preventivo nem curativo para a Doença de Alzheimer, a fisioterapia tem seu papel importante no tratamento, utilizando-se de vários métodos, sendo um deles o método Kabat 42.

A avaliação fisioterapêutica dependerá do comportamento que paciente vai apresentar, considerando o estagio da doença. No inicio da doença os itens a serem avaliados são: amplitude articular, força muscular, alterações posturais e capacidade pulmonar. Elementos como coordenação motora, equilíbrio, autopercepção, marcha, habilidade, imagem corporal e as funções a qual o indivíduo exerce durante a vida diária deve ser valorizada e avaliada de forma cautelosa. Nas fases mais tardias por conta do comprometimento físico ser bem maior a avaliação de mobilidade é feita com movimentos passivos, nessa fase a avaliação pulmonar torna-se mais críticas ${ }^{42,43}$.

A partir da segunda fase da DA, o portador começa a apresentar importantes deficiências motoras e já apresenta dificuldade na realização de AVDs e alteração da marcha. O tratamento fisioterapêutico passa a ter grande importância para retardar a progressão das perdas motoras, evitar encurtamentos e deformidades e incentivar a independência do doente ${ }^{42,43}$.

É imprescindível deixar claros os objetivos da fisioterapia tanto para a equipe como para os familiares e o paciente a ser tratado, facilitando, a aceitação ao tratamento proposto ${ }^{44}$. Exercícios físicos podem ser usados como estratégia preventiva, já que alguns estudos mostram que a prática regular é importante para manter o equilíbrio, a força e a cognição em pacientes com DA. Além disso, exercício está associado com menor prevalência e incidência de demência (32\%), bem como de declínio cognitivo. Estudos mostraram que um programa de exercícios generalizados (resistência, mobilidade e coordenação) pode diminuir a deterioração no desempenho das AVD significativamente, aumentando a capacidade funcional global e a habilidade para desempenhar as AVD 2, 41. 
O biomédico como profissional da saúde pode auxiliar nas áreas que envolvam avaliações da qualidade de vida deste paciente, criar estratégias educativas para os familiares e cuidador que assumirão o papel de cuidar, e assim suprir a incapacidade funcional, temporária ou definitiva de seu familiar ${ }^{4}$.

Em conjunto com outros profissionais da saúde, o biomédico deve desenvolver estratégias de prevenção, promoção e intervenção na busca de uma velhice bem-sucedida junto aos familiares e cuidadores, interagindo e orientando-os para que não sejam acometidos por desgaste físico e emocional. Orientar e transmitir o conhecimento sobre as características da doença de Alzheimer, seu curso e como lidar com problemas diários vivenciados pelo idoso, pois muitos cuidadores não possuem um suporte de conhecimento necessário para o cuidado e tornam-se sobrecarregados $41,36,45,46$. Podendo ainda colaborar na pesquisa de novos tratamentos baseando-se nas informações sólidas existentes sobre o curso da doença e assim desvendar as causas que levam à morte neuronal na Doença de Alzheimer ${ }^{2,} 26$.

\section{CONCLUSÃO}

Apresar de não existir cura para a Doença de Alzheimer, pois o próprio diagnostico da doença é complexo, o exercício físico vem aparecendo ser uma alternativa, a fisioterapia no tratamento da doença de Alzheimer vem demostrando ótimos resultados, pois a pratica regular ajuda a conservar a funcionalidade, mantendo força, equilíbrio e preservando a cognição, associado a pratica regular de exercícios a menor prevalência e incidência da demência.

A fisioterapia independente da modalidade utilizada para terapêutica, desde que avaliados os pacientes e elaborado a partir da avaliação um plano de tratamento adequado e individualizado para os portadores da doença de Alzheimer, de acordo com a funcionalidade e o estagio da doença, com objetivo de minimizar as dificuldades e retardar a progressão da doença.

A doença de Alzheimer deixa claro a necessidade de se trabalhar estratégias de prevenção, promoção de saúde, orientação sobre essa doença para familiares 
de indivíduos e para a comunidade, contado com a Biomedicina, na busca de um envelhecimento saudável, junto aos familiares e cuidadores, interagindo e orientando-os. Além das pesquisas para desvendar as causas da doença e possível tratamento, ou uma abordagem mais positiva para com o paciente, baseada nas experiências reais de convívio.

\section{REFERÊNCIAS}

1 Veras R. Envelhecimento populacional contemporâneo: demandas, desafios e inovações. Rev. Saúde Pública. 2009; 43 (3):548-554.

2 Garrido R, Menezes PR. Impacto em cuidadores de idosos com demência atendidos em um serviço psicogeriátrico. Rev. Saúde Pública. 2004; 38 (6):835-841.

3 Lemos CA, Hazin I, Falcão JTR. Investigação da memória autobiográfica em idosos com Demência de Alzheimer nas fases leve e moderada. Estudos de Psicologia. 2012; 17(1):135-144.

4 Facure NO, Castro LAG, Menezes MCL. Doença de Alzheimer: relação entre o tempo de doença e seu estadiamento. Arq. Neuro-Psiquiatr. 1993; 51(2):175-178.

5 Rodrigues J, Franco ML. Case study: systematization of nursing care for an elder with mild dementia and his caregiver. [Monography]. [Brasília]: Universidade Católica de Brasília; 2012. 182 p.

6 Guerreiro T, Caldas, CP. Memória e demência: (re)conhecimento e cuidado. Rio de Janeiro: Unati, 2001. 204p.

7 Bolognesi ML, Rosini M, Andrisano V, Bartolini M, Minarini A, Tumiatti V, Melchiorre C. MTDL design strategy in the context of Alzheimer's disease: from lipocrine to memoquin and beyond. Curr Pharm. 2009; 15: 601-613. 
Artigo Original

Fisiopatologia

8 Morales GI, Farias GG, Maccioni BRB. La neuroinflamación como factor detonante del desarrollo de la enfermedad de Alzheimer. Rev. chil. neuro-psiquiatr. 2010; 48(1):49-57.

9 Fernandez, LL; Coutinho, LM. Alterações encontradas em cérebros de indivíduos acima de 65 anos e sua correlação com a Demência de Alzheimer. Arq. NeuroPsiquiatria. 1993; 55(2): 298-304.

10 Cavalcanti JLS, Engelhardt E. Aspectos da Fisiopatologia da Doença de Alzheimer Esporádica. Rev Bras de Neurologia. 2012; 48(4): 21-29.

11 Cruz MN, Hamdan AC. O Impacto da Doença de Alzheimer no Cuidador. Psicologia em Estudo. 2008; 13 (2):223-229.

12 Sereniki A, Vital MABF. A Doença de Alzheimer: aspectos fisiopatológicos e farmacológicos. Rev Psiquiatr. Rio Grande do Sul. 2008; 30(1) suppl.. ISSN 01018108. http://dx.doi.org/10.1590/S0101-81082008000200002.

13 Dias FLC. Perfil Clínico e Autonômico de Pacientes com Doença de Alzheimer e Demência Mista. Rev. Assoc. Médica Bras. 2013; 59(5):455-441.

14 Fonseca SR. Perfil Neuropsiquiátrico na Doença de Alzheimer e na Demência Mista. Jorn. Bras. Psiquiatr. 2008; 5(2):117-121.

15 Peçanha MAP, Neri VC. Estudo Neuropatológico e Funcional da Doença de Alzheimer. Rev. Científ. Faculd Med Campos. 2007; 2(1):1980-7813.

16 Engelhardt E. Tratamento da Doença de Alzheimer Recomendações e sugestões do Departamento Científico de Neurologia Cognitiva e do Envelhecimento da Academia Brasileira de Neurologia. Arq.Neuro-Psiquiatr.2005; 63(4):1104-1112. 
17 Burla C. Panorama Prospectivo das demências no Brasil: Um enfoque demográfico. Ciênc. Saúd. Colet. 2013; 18(10): 2949-2956.

18 Qiu C, De Ronchi D, Fratiglioni L. The epidemiology of the dementias: an update. Curr Opin Psychiatry. 2007; 20(4):380-385.

19 Lobo A. Prevalence of dementia and major subtypes in Europe: A collaborative study of population-based cohorts. Neurologic Diseases in the Elderly Research Group. Neurology. 2000.

20 - Aprahamian I, Martinelli JE, Yassuda MS. Doença de Alzheimer: Revisão da epidemiologia e diagnóstico. Rev. Soc. Bras. Clínica Médica. 2009; 7(1):27-35.

21. Silva KCA, Ribeiro PCC, Lourenço RA. Epidemiologia das Demências. Rev. Hosp. Univ. Pedro Ernesto, UERJ; 2008; 7(2):46-51.

22. The International Federation of Alzheimer's. World Alzheimer Report 2009. Executive Summary.

23 De Lima Torres KC, Dos Santos RR, Mapa FC, Lanna F, De Moraes ENDM, Silva MA R. Biomarcadores na doença de Alzheimer. Belo Horizonte:2012.

24 Starling, DSV. Investigação de biomarcadores diagnósticos para a doença de Alzheimer no líquido cefalorraquidiano, na saliva e na mucosa oral; 2012; 111-113.

25. Ribeiro CF, Doença de Alzheimer: a principal causa de demência nos idosos e seus impactos na vida dos familiares e cuidadores. [Monografia].[ elo Horizonte] Enfermagem, Universidade Federal de Minas Gerais, 2010.1: 1-60

26 Gemelli T, Andrade RB, Castro AL. Estresse Oxidativo Como Fator Importante na Fisiopatologia da Doença. Revista Uniara. 2013;16 (1):67-78. 
Artigo Original

Fisiopatologia

27 Associação Médica Brasileira, Agência Nacional de Saúde Suplementar. Diretrizes Clínicas na Saúde Suplementar: Doença de Alzheimer - diagnóstico. 2011.

28 Viegas FPD. Doença de Alzheimer: Caracterização, Evolução e Implicações do Processo Neuroinflamatório. Rev. Vital de Química. 2011; 3(4): 286-306.

29 BRASIL. Portaria no 491 de 23 de setembro de 2010. Aprova o protocolo clínico e diretrizes terapêuticas para a doença de Alzheimer.

Forlenza OV. Tratamento farmacológico da doença de Alzheimer. Rev. Psiquiatr. Clínica. 2005; 32(3):137-148.

31 Lima FB. Estudo sobre a ocorrência de micronúcleos e alterações nucleares em indivíduos com a Doença de Alzheimer. Rev. Ciências Médicas e Biológicas. 2012; $11(1): 23-26$.

32 Souza ND, Silva CR, Silva, VB. Donepezil no tratamento da Doença de ALZHEIMER. Estudos Goiânia. 2014; 41(4): 755-766.

33 Forlenza, O. V. Tratamento farmacológico da doença de Alzheimer. Revista de psiquiatria clínica. 2005; 32(3)137-148.

34 Pinto, MCX, Resende RR. Excitotoxicidade e Doenças Neurológicas; 2014; 2(4): $240 p$.

35 The WHOQOL Group. The World Health Organization quality of life assessment: position paper from the world health organization, 1995. Soc Sci Med. 1995;41(1):1403-1409.

36 Brasil MC. Andrade C. Reconfiguração de campo do familiar cuidador do portador de Alzheimer. Psicol. Estud; 2013; 18(4) 324-331. 
37 Cordeiro J, Castillo BLD, Freitas CS, Gonçalves, MP. Efeitos da atividade física na memória declarativa, capacidade funcional e qualidade de vida em idosos. Rev. bras. geriatr. gerontol. 2014; 17(3): 189-195.

38 Guedes DV, Barbosa, AJ, Magalhães NC. Qualidade de vida de idosos com declínio cognitivo: auto e heterorrelatos. Aval. psicol. 2013; 12(1):442-449.

39Leite CDSM, Menezes TLM, Lyra VV, Araújo CMT. Conhecimento e intervenção do cuidador na doença de Alzheimer: uma revisão da literatura. J. bras. psiquiatr.2014; 63(1): 212-220.

40 Pereira KCR, Alvarez AM, Traebert JL. Contribuição das condições sociodemográficas para a percepção da qualidade de vida em idosos. Rev. bras. geriatria gerontologia. 2011; 13(3): 201-211.

41 Zidan M, Arcoverde C, Araújo NB, Vasques P, Rios A, Laks J, Deslandes A. Alterações motoras e funcionais em diferentes estágios da doença de Alzheimer. Rev. psiquiatr. Clín. 2012; 39(5)12.

42 Carvalho KR, Cabral RMC, Gomes DAGS, Tavares AB. O método Kabat no tratamento fisioterapêutico da doença de Alzheimer Adriano Bueno revista Kairós, 2008; 11(2):181-195.

43 Melo MA, Driusso P. Proposta Fisioterapêutica para os cuidados de Portadores da Doença de Alzheimer. Envelhecimento e saúde. 2006; 12 (4):11-18.

44 Marcucci, FCl. O papel da fisioterapia nos cuidados paliativos a pacientes com câncer. Rev Bras Cancerologia. 2006; 5,(1): 67-77.

45 Woellner SS, Araújo AGS, Risso PR, Junior HH. Estudo comparativo da mobilidade orientada pelo desempenho em idosos com e sem doença de Alzheimer. Revista Brasileira de Medicina: 2010; 69(11). 
Artigo Original

Fisiopatologia

46 Faria, I M. Qualidade de Vida das Pessoas com Alzheimer: estudo comparativo de dois contextos residencias. 2010. Dissertação (Mestrado) - Curso de Psicologia, Instituro Universitario Pispa, Portugal. 81 p. 